The final section related to the mechanical properties of ionic solids, as affected by point dofect. The introductory lecture by Prof. Pratt showed cloarly that two main categories of effects could be considered; the direct effects of defects or their aggregates, and the indirect effect of tho interaction of the defects with pre-existing dislocation or dislocations formed by mechanical deformation. It appears that in some circumstances a main effect may be the indirect pinning of dislocations by their electrostatic or stress interaction with ro-oriontable dipole-type defects. A short paper on sodium chloride with controlled concentration of divalent cations supported this view. The nature of the relaxation of dipoles formed by trapped defects was discussed in group theoretical terms in the next paper, and it was shown that except for very short times a single relaxation time appropriate to noarest neighbour orientations of the defect is reasonably accurate.

The effects of irradiation on the plastic properties of sodium chloride showed a rise in yield stress, a tendency to smaller slip-step heights and to simultaneous slip on equivalent planes with suppression of kinking. None of the changes was related to $F$-band colour, but $V$ band and interstitial density were not checked. The effects observed in the Youngs modulus of polycrystalline graphite after low-temperature electron irradiation appeared to be due to the pinning of dislocations, but no detailed mechanism could be proposed.

Electron microscope examinations have been made on a number of deformed and defective crystals. The development of oxygen deficiency in titanium dioxide helps the annealing out of stacking faults by a mechanism which may well involve the dissolution of titanium interstitial atoms, after condensation of oxygen vacancies has eliminated planes of oxygen. The titanium interstitials aro in fact identified by colour studies. Other dislocation roactions have also been observed. A separate group reported an increase in optical absorption associated with an increase in titanium interstitial content after hightemperature plastic deformation of an already oxygendeficient titanium dioxide crystal, and also attributed this to either stacking fault elimination or to dislocation climb. In view of the lack of structure in the optical absorption investigations, several comments were made to the effect that an increase in titanium interstitial content could not yet be accepted as proved. Electron microscope examinations were also reported on plastically deformed TiO, and annealing investigations pointed to an activation energy of some $5 \cdot 5 \mathrm{eV}$ for atomic mobility.

To sum up, this was a good conference to be at. It brought together groups of people who meet too seldom, and showed again the unity of the physical sciences. It was the right size-about 120 people-and the programme was not so packed that it was impossible to find a moment to talk to most of those one meant to. The wisdom of holding the conference during university term, when many people found it hard to be away for three days, and when it was not possible to use the lecture theatres of a university, may be queried. A proper lecture room is really quite important, though not nearly so important as the programme, content and participants. I am sure the success of this meeting will encourage the British Ceramic Society to hold more meetings of similar kind to bring these neglected substances under regular scientific review.
W. M. LOMER

\title{
MECHANISMS OF ADSORPTION FROM SOLUTION
}

$\mathrm{A}^{\mathrm{s}}$ $\mathrm{N}$ informal discussion of the Faraday Society under the above title was held in the Department of Mechanical Engineering, Imperial College of Science and Technology, during September 19-20, by kind permission of Prof. O. A. Saunders. The meoting was attended by 144 people, 81 of whom came from industrial organizations and 63 from academic institutions. There were 16 overseas visitors.

Sir Eric Rideal opened the meeting and, as the first item, Dr. J. J. Kipling (University of Hull) gave a review of the present position in this subject. After outlining the main historical periods, and the progress which was made in each, Dr. Kipling discussed at some length the problems encountered when, instead of an adsorbing vapour, we have to deal with a two (or more) component liquid system. The basic equation relating changes in the surface and bulk mole fractions of the components was given, and its relation to the Gibbs surface excess and the individual adsorption isotherms for the components was described. The use of adsorption from solution for measurement of surface area was examined critically, and, in particular, the difficulty of selecting an appropriate cross-sectional area for the adsorbate molecule was stressed. The difficulty of satisfactorily taking into account localized site adsorption was also discussed. Finally, Dr. Kipling mentioned the question of chemisorption of molecules from solution and foresaw the problem well known in vapour adsorption of distinguishing between chemically and physically adsorbed molecules.

The first paper was given by Prof. D. H. Everett (University of Bristol) and was entitled "Thermodynamic Models of Adsorption from Solution". The adsorption of the components of a binary liquid mixture on to a uniform surface was described in statistical mechanical terms and a new definition of the activity coefficients of the components in the adsorbed layers was proposed.
Prof. Everett showed that the "isotherm of concentration change" may be markedly dependent on the nature of the solution concerned, for example, whether it is a regular solution, or contains molecules of different size. The use of adsorption from solution for the determination of specific surface areas was mentioned and it was suggested that useful results could be obtained from measurements of adsorption from mixtures of molecules of approximately the same size (for example, cyclohexane + benzene).

This was followed by a paper on the "Thickness and Structure of Layers of High Polymers adsorbed from Solutions to Solid Surfaces", by F. Rowland, R. Bulas and Prof. F. R. Eirich (Polytochnic Institute, Brooklyn, New York). The paper was given by Prof. Eirich, who explained the difficulties and uncertainties of investigating the nature of polymer adsorption and then presented results obtained principally by observing the change in flow of liquids through sintered glass disks of known pore size distributions and surface areas. From an analysis of the hydrodynamics of the system, the change in effective radius of the capillaries resulting from polymer adsorption was estimated. Several polymers in different non-aqueous solvents have been examined and it was shown that the film thicknesses were of the same order of magnitude as the diameters of the free coils in solution. A detailed discussion was given of the nature of the adsorption isotherms, of their reversibility and of the influence of good and bad solvents.

A contribution to the investigation of the adsorption of ionized polymers was made by J. B. Craig and Dr. P. Meares (University of Aberdeen) under the title "The Adsorption of Polyacrylates at a Charged Mercury-Salt Solution Interface". Experimental data had been obtained from electrocapillarity investigations, and from derived thermodynamic relations, the surface excess concentrations of polyanions, counter-ions and co-ions 
wore estimated. The plot of surface excess of polyanion against increasing bulk concentration showed a maximum followed by a decrease to an almost constant value. A detailed model was proposed to account for this and related phenomena which was based on configurational changes of the polyanions normal and parallel to the surface. From the calculated co-ion surface excesses an estimate was made of the thicknoss of the adsorbed polyion film; this appeared, in some cases, to be greater than one monolayer.

On the following morning, under the chairmanship of Dr. J. A. Kitchener, Dr. C. H. Giles (University of Glasgow) presented a paper on "The Association of Adsorbed Organic Solutes". The adsorption of a number of organic molecules, many of them dyes, on to a variety of adsorbents was described and compared with the surface area indicated by $p$-nitrophenol adsorption. The differences relative to $p$-nitrophenol were expressed as 'coverage factors', and it was shown that these factors bore a simple relation to the molecular weight of the adsorbate. The 'coverage factors' were suggested to arise from the adsorption of the organic molecules as an aggregate or micelle. Some direct evidence for such a phenomenon was presented.

"Adsorption of Cyanine Dyes at Silver Halide Surfaces" was the title of a paper by Dr. J. F. Padday (Kodak, Ltd.). Tho surface areas of specimens of silver chloride, bromide and iodide were determined from argon and benzene vapour adsorption. It was deduced that under conditions of close packing of the cyanine dyes at the crystal surfaces the area per molecule of a given dye was different for the various halides. From this evidence and from moasurements of spectral reflectance the manner in which the substrate affected the state of the dye was discussed.

The chairman for the final session was Prof. F. C. Tompkins. In the first part Dr. I. T. Smith (Paint Research Station, Toddington) gave a paper on "An Infrared Spectroscopic Study of the Adsorption of Carboxylic Acids on Titanium Dioxide Pigments". After adsorption equilibrium had been ostablished in each case, for a variety of carboxylic acids in benzene in contact with soveral different samples of titanium dioxide, the oxide was made into disks and examined by absorption spectroscopy. The results suggested that the acids were bound to the oxides, either through a carboxylate ion, or by means of hydrogen bonds. Both types of bonding could usually be found for a given pigment. It was found that on washing the oxide with its adsorbed molecules in pure solvent the hydrogon bonded molecules, but not perceptibly tha ions, were removed. Immersion in the original acid solution restored the specimen to its original state.

The meeting ended with an extended discussion on the adsorption of polar molecules on to metal oxides. Dr. J. J. Kipling and Dr. E. H. M. Wright mentioned that stsaric acid molecules adsorbed from benzene on to silica lay flat on the surface, but on alumina and titanium dioxide were approximately vertical. They also showed that for stearic acid on titanium dioxide and alumina the adsorption was close to that expected for a localized adsorption on to lattice points. Dr. D. A. Haydon and P. T. Dawson (University of Cambridge) described the effectively irreversible adsorption at $20^{\circ} \mathrm{C}$ of formic acid from the vapour phase and stearic acid from benzene on three different dry activated rutiles. The possible reasons for a slightly lower adsorption, in all cases, of stearic acid rolative to formic were discussed. Both stearic and formic acids gave fractional covorages relative to nitrogen of approximately 0.5 . Dr. G. D. Parfitt and I. J. Wiltshire (University of Nottingham) discussed the adsorption of a homologous series of aliphatic aleohols from xylene on to a pure rutile. The amount of alcohol adsorbed showed a strong dependence on chain-length, being a minimum at octanol. Prof. Everett commented that such behaviour might be expected from his theoretical treatment of adsorption. The important part played by adsorbed water on the rutile surface in the adsorption process was also stressed.

During the meoting it was announced that it was proposed to hold a further meeting during September 17-18, 1964, in the Chemistry Department, University of Bristol, on the subject, "The Electrical Double Layer in Surface and Colloid Chemistry".

R. H. OtTewili.

\section{METABOLISM AND PHYSIOLOGICAL SIGNIFICANCE OF LIPIDS}

\begin{abstract}
IPID biochemistry is one of the scientific topics which Lave become prominent during the past fifteen years, due both to major technical advances easing their study and to fundamontal observations showing their importance. Thus only part of the fiold could bo surveyed at the advanced study conference on the metabolism and physiological significance of lipids, held at Cambridge during September 16-21, 1963, and supported by the Scientific Affairs Division of NATO, although about 150 participants (from institutions in sixteen countries, of which six do not belong to NATO) hoard 38 formal discourses given at nine day-time sessions, besides two informal ovening symposia, and were admirably provided by the organizers, Drs. R. M. C. Dawson and D. N. Rhodes (Great Britain), with time and electronic encouragement for free discussion.

The first major topic was lipid biosynthesis. Prof. S. J. Wakil (United States) began by describing how animals form long-chain fatty acids. First, a reductive synthesis of saturated acids from malonyl and acctyl coonzymo $A$ gives mainly palmitate; it, or longer-chain acids, can then be oxidativoly desaturated. Secondly, in the most recently examined process, acetyl coonzyme $A$ providos two carbon atoms for the reductive chain-lengthening of saturated or unsaturated acids; thus palmitoleate gives cis-vaccenate, now known to be an important component of 'oleate'.
\end{abstract}

According to Prof. K. Bloch (United States), some organisms form unsaturated acids anaerobically by the dehydration of hydroxy acids, but most do so aerobically. Only green plants can make linoleate, which is then further dehydrogenated via $\alpha$-linolenate; or, in animals, via $\gamma$-linolenate. The route used and the type of compound lipid produced by green euglenids depend on whether they are functioning as plants or animals. Photosynthetic bactoria do not produce polyunsaturated fatty acids, which may therofore be specifically concerned in plants' oxygen-yielding type of photosynthesis.

Prof. B. Shapiro (Israel) said that synthesis of triglycerides by the labile enzyme systems of liver preparations in vitro is greatly assisted by lecithin or by serum proteins, and closoly dopendent on the concentration of $\alpha$-glycerophosphate, which varies enormously under different physiological conditions in vivo and may therefore control the process.

Dr. G. Popjak (Great Britain) then showed how deutorium-labelled mevalonate and tritium-labelled pyridine nucleotides of known absolute configuration have been used to establish the precise stereochemical specificity of the reactions whereby mevalonate is converted via its pyrophosphate and isopentenyl, geranyl and farnesyl pyrophosphates into squalene, the precursor of steroids. 